\title{
Some general problems on the number of parts in partitions
}

\author{
by
}

\section{B. Richmond (Waterloo, Ont.)}

1. Introduction. Let $A=\left\{a_{1}, a_{2}, \ldots\right\}, a_{1}<a_{2}<\ldots$ denote a sequence of positive integers. We consider the following four partition functions. Let $p_{A}(m, n)$ denote the number of solutions of

$$
n=x_{1}+x_{2}+\ldots+x_{m}, \quad x_{1} \leq x_{2} \leq \ldots, x_{i} \in A,
$$

and let $\bar{p}_{A}(m, n)=p_{A}(m, 1)+p_{A}(m, 2)+\ldots+p_{A}(m, n)$. Let $q_{A}(m, n)$ denote the number of solutions of

$$
n=x_{1}+x_{2}+\ldots+x_{m}, \quad x_{1}<x_{2}<\ldots, x_{i} \in A,
$$

and let $\bar{q}_{A}(m, n)=q_{A}(m, 1)+q_{A}(m, 2)+\ldots+q_{A}(m, n)$.

For each of these functions P. Turán [15] proposed the problem of finding a general class of sequences $A$ and a suitable $f(n, A)=f(n)$ so that for almost all such partitions,

$$
m=(1+o(1)) f(n), \quad n \rightarrow \infty .
$$

Here "almost all" means with at most $o\left(p_{A}(n)\right)$ exceptions where $p_{A}(n)=$ $p_{A}(1, n)+p_{A}(2, n)+\ldots+p_{A}(n, n)$ for example.

When $A$ is the sequence of all natural numbers Erdös and Lehner [3] showed that for $p_{A}(m, n)$,

$$
\left|m-\frac{\sqrt{6}}{2 \pi} n^{1 / 2} \log n\right|<n^{1 / 2} w(n)
$$

holds for almost all partitions of $n$ provided $w(n) \uparrow \infty$ arbitrarily slowly, hence for this function $f(n)$ equals $(2 \pi)^{-1}(6 n)^{1 / 2} \log n$. Erdös and Lehner (l.c.) show that when $A$ is the sequence of natural numbers then $f(n)=$ $2 \cdot \pi^{-1} \cdot 3^{1 / 2} \log 2 \cdot n^{1 / 2}$ for $q_{A}(m, n)$. Erdös and Turán [4] treated $q_{A}(m, n)$ and $\bar{q}_{A}(m, n)$. They found a density requirement on $A$ that allows the de- 
termination of $f(n)$ for $\bar{q}_{A}(m, n)$. They assumed that

$$
A(x)=\sum_{a_{i} \leq x} 1
$$

satisfies

$$
\lim _{x \rightarrow \infty} x^{-\alpha} \log ^{\beta} x \cdot A(x)=\gamma, \quad 0<\alpha \leq 1, \beta \text { real, } \gamma>0,
$$

and found that almost all of the partitions counted by $\bar{q}_{A}(m, n)$ consist of

$$
(1+o(1)) \gamma_{1} n^{\alpha /(\alpha+1)} \log ^{-\beta /(\alpha+1)} n
$$

parts where $\gamma_{1}$ is a complicated constant. They also found that if $A(x)$ satisfies the relation

$$
A(x)=\gamma_{2} x^{\alpha} \log ^{-\beta} x\left(1+O\left((\log x)^{-1}\right)\right), \quad 0<\alpha \leq 1, \beta \text { real, } \gamma_{2}>0,
$$

and moreover if

$$
\log q_{A}(n)>\gamma_{3} n^{\alpha /(\alpha+1)} \log ^{-\beta /(\alpha+1)} n\left(1-\frac{\log ^{-1 /(2 \alpha+2)} n}{\log \log n}\right)
$$

where

$$
\gamma_{3}=\alpha^{-\alpha /(\alpha+1)}(1+\alpha)^{1+\beta /(\alpha+1)}\left(\gamma_{2}\left(1-2^{-\alpha}\right) \zeta(\alpha+1) \Gamma(\alpha+1)\right)^{1 /(1+\alpha)},
$$

then almost all of the partitions counted by $q_{A}(m, n)$ consist of

$$
\gamma_{1} n^{\alpha /(\alpha+1)} \log ^{-\beta /(\alpha+1)} n\left(1+O\left(\log ^{-1 /(4 \alpha+4)} n\right)\right)
$$

parts.

In this paper we determine the asymptotic behaviour of $p_{A}(m, n)$ and $\bar{p}_{A}(m, n)$ for those $m$ near the values of $m$ which maximize each function. Our density requirements are weaker than those of Erdős and Turán. For $p_{A}(m, n)$ and $\bar{p}_{A}(m, n)$ instead of (1.1) we suppose that either

$$
\lim _{i \rightarrow \infty} \frac{\log a_{i}}{\log i}=s \quad \text { exists }
$$

or $\log a_{i}=O(\log i)$ and

$$
A(2 x)=O(A(x)) \quad \text { as } x \rightarrow \infty .
$$

(This is a W. Schwarz condition [12].) We impose no additional condition for $\bar{p}_{A}(m, n)$. Turán [15] describes the investigations of $p_{A}(m, n)$ and $\bar{p}_{A}(m, n)$ as incomplete. We find that for $\bar{p}_{A}(m, n)$ when (1.5) or (1.6) holds a necessary and sufficient condition for $f(n)$ to exist is that $\sum a_{i}^{-1}$ diverges. We shall express our formulas in terms of the unique solution of a certain equation, however, Mellin transform techniques [7] allow us to derive the formulas (1.2) and (1.4). We shall see that $p_{A}(m, n)$ and $\bar{p}_{A}(m, n)$ are strictly increasing with $m$ to a maximum achieved by at most two consecutive values of $m$ and 
then strictly decreasing under quite weak conditions on $A$, at least for $m$ near the maximum.

For the sake of completeness and also to introduce some required notation we sketch the results of Haselgrove and Temperley [5] concerning $p_{A}(m, n)$. Let

$$
G(u, t)=\sum_{m=0}^{\infty} \sum_{n=0}^{\infty} p_{A}(m, n) u^{m} t^{n}=\prod_{r=1}^{\infty}\left(1-u t^{a_{r}}\right)^{-1} .
$$

We write $u=\exp (-v), t=\exp (-w)$ and then $v=\alpha w$. Then if $G(u, t)=$ $g(\alpha, w)$ we have

$$
g(\alpha, w)=\prod_{r=1}^{\infty}\left(1-\exp \left\{-\left(a_{r}+\alpha\right) w\right\}\right)^{-1} .
$$

Note that if $\operatorname{Re} w>0$ the product for $g(\alpha, w)$ is absolutely convergent. Hence $g(\alpha, w)$ is a regular function of $\alpha$ with poles at the points $\alpha=-a_{r}+2 k \pi i w^{-1}$ for any fixed value of $w$. If $\sum a_{r}^{-2}$ converges we may define

$$
K(\alpha)=\prod_{r=1}^{\infty}\left(1+\alpha / a_{r}\right)^{-1} e^{\alpha / a_{r}} .
$$

$K(\alpha)$ is a regular function with poles at the points $\alpha=-a_{r}$. Note that $g(\alpha, w) / K(\alpha)$ is a regular function of $\alpha$ for $|\alpha|<\operatorname{Re}(2 \pi / w)$. Since both $g(\alpha, w)$ and $K(\alpha)$ are nonzero the same holds for $\log (g(\alpha, w) / K(\alpha))$.

Haselgrove and Temperley [5] assume that $\sum a_{i}^{-2}$ converges and that several conditions are satisfied by the function $\psi(w)$ where

$$
\psi(w)=\sum_{r=1}^{\infty} e^{-a_{r} w}, \quad w=\xi+i \eta, \xi>0 .
$$

For example, they suppose that

$$
\left\{\psi^{\prime}(\xi)\right\}^{2}<\theta \psi(\xi) \psi^{\prime \prime}(\xi) \quad \text { ((iii) in their paper) }
$$

for some fixed $\theta<1$ and for $\xi$ sufficiently small. We replace the HaselgroveTemperley conditions (ii), (iii) and (iv) by either (1.5) or (1.6). They also suppose ((v) in their paper) that for sufficiently small $\xi,|\psi(w)|<\theta \psi(\xi)$ in the region $\xi \Delta \leq|\eta| \leq \pi$ for any fixed $\Delta$ and some $\theta<1$ depending only on $\Delta$. This is a difficult condition to work with (they state that it does not hold when $A$ is the sequence of primes for example and must replace it in this case by deep results of Vinogradov and Linnik concerning Goldbach's conjecture) so we replace this condition by the following arithmetical condition: Recall that Bateman and Erdös [2] say that a sequence $A$ of integers, not necessarily positive, has property $P_{k}$ if there are more than $k$ elements in $A$ and if we remove an arbitrary subset of $k$ elements from $A$ the remaining elements have greatest common divisor unity. We say $A$ has property $Q_{k}$ 
if it has more than $k$ elements and if we remove an arbitrary subset of $k$ elements from $A$ the remaining elements are not in the same residue class modulo $m$ for any $m>1$.

Let us consider some examples. If $a_{i}=i^{k}$ then $A=\left\{a_{i}\right\}$ will have property $Q_{k}$ for all $k \geq 0$. To check this we need only note that $i^{k}$ is congruent to 0 and 1 infinitely often for every modulus $m>1$. If $a_{i}=p_{i}$, the $i$ th prime, then $A$ will have property $Q_{0}$ but not $Q_{1}$ since deleting 2 leaves us with a set of odd numbers. We shall obtain an asymptotic formula for $p_{A}(m, n)$ when $A$ is the set of primes, but we expect that $p_{A}(m, n)$ is not uniformly increasing then decreasing near its maximum as a function of $m$.

Suppose $A$ has property $Q_{k}$ but not $Q_{k+1}$. Then there will be a set $\bar{A}$ of $k+1$ elements such that the elements of $A-\bar{A}$ all lie in one residue class. Furthermore, for each $a$ in $\bar{A}$ we can find a set, $A_{a}$, of $k+1$ elements of $A$ such that if $\theta \notin \mathbb{Z}$ then $\varphi-a \theta \neq \varphi-b \theta+l, l \in \mathbb{Z}$, for any $b \in A_{a}$ (see the proof of (2.31)). Let

$$
\widetilde{A}=\bar{A} \cup \bigcup_{a \in \bar{A}} A_{a} .
$$

We can also write every element, $b$, of $A-\widetilde{A}$ in the form $b=m d+r$. Let $B$ denote the sequence of $m$ defined in this way, or

$$
B=(A-\widetilde{A}-r) / d .
$$

We let

$$
\Psi(\xi)=-\sum_{r} \log \left(1-e^{-\xi a_{r}}\right), \quad m_{0}(\xi)=\sum_{r}\left(e^{\xi a_{r}}-1\right)^{-1},
$$

and

$$
F(y)=\frac{1}{2 \pi i} \int_{-i \infty}^{i \infty} K(\alpha) e^{\alpha y} d \alpha .
$$

Our first theorem, proved in Section 2, is

TheOREM 1.1. Suppose $A$ has property $Q_{k}, k \geq 0$, and satisfies either (1.5) or (1.6). Suppose the $B$ of (1.10) has property $Q_{l}, l \geq 0$. Then as $n \rightarrow \infty$,

$$
\begin{aligned}
\Delta_{m}^{r} \Delta_{n}^{s} p_{A}( & m, n) \\
= & \xi^{r+s+1} F^{(r)}\left(\left(m-m_{0}\right) \xi\right) p_{A}(n) \\
& +O\left(\xi^{r+s+1} \psi^{-1 / 8}(\xi) p_{A}(n)\right)+O\left(\xi^{k+l-1 / 2-\varepsilon} p_{A}(n)\right) \\
& +O\left(\xi^{k+3 / 2-\varepsilon} p_{A}(n)\right), \quad \varepsilon>0,
\end{aligned}
$$

where $\xi$ is a root of $\Psi^{\prime}(\xi)+n=0$ and $\Delta_{m}$ and $\Delta_{n}$ denote the difference operators, so that 


$$
\Delta_{n} p_{A}(m, n)=p_{A}(m, n)-p_{A}(m, n-1), \quad \text { etc. }
$$

Also, if $A$ has property $P_{k}$ of Bateman and Erdös [2], $k \geq 0$, it is proved in Richmond [9] that $p_{A}(n)$ satisfies

$$
p_{A}(n)=\frac{1}{\sqrt{2 \pi}}\left(\Psi^{\prime \prime}(\xi)\right)^{-1 / 2} \exp \{\Psi(\xi)+n \xi\}(1+O(\xi)) .
$$

In particular, if $k \geq 1$,

$$
p_{A}(m, n) \sim \xi F\left(\left(m-m_{0}\right) \xi\right) p_{A}(n)+o\left(\xi p_{A}(n)\right),
$$

and if $k \geq 2$,

$$
\Delta_{m} p_{A}(m, n) \sim \xi^{2} F^{\prime}\left(\left(m-m_{0}\right) \xi\right) p_{A}(n)+o\left(\xi^{2} p_{A}(n)\right) .
$$

We also prove

TheOREM 1.2. The results in Theorem 1.1 hold for $\bar{p}_{A}(m, n)$ with $k$ replaced by $k+1$ in the error terms. The second last result of Theorem 1.1 holds for any $A$ having property $Q_{0}$ and satisfying either (1.5) or (1.6) with $p_{A}(n)$ replaced by $\sum_{l \leq n} p_{A}(l)$.

The distribution $F(y)$ has many remarkable properties unknown to Haselgrove and Temperley when they wrote their paper. A beautiful description of the theory of $F(y)$ is given in the book of Hirschman and Widder [6]. There it will be found for example that I. J. Schoenberg in a series of papers starting in 1947 showed that for $n=0,1, \ldots, F^{(n)}(y)$ has exactly $n$ changes of sign and that $-\log F(y)$ is convex. Hence $F(y)$ will have a unique maximum and if $A$ satisfies property $Q_{1}$ and (1.5) or (1.6) then $p_{A}(m, n)$ will either have a unique maximum for large $n$ as a function of $m$ or the maximum will be achieved at exactly two consecutive values of $m$. This was first conjectured by Auluck, Chowla and Gupta [1] when $A$ is the sequence of all natural numbers and first proved by Szekeres [13], [14] and Haselgrove and Temperley [5]. (Szekeres proved a good deal more, namely that $p_{A}(m, n)$ is unimodal in this case for large $n$.) Haselgrove and Temperley also prove the analogous result when $A$ is the sequence of squares and conjecture the same for the sequence of $k$ th powers. Theorem 1.2 establishes this result in rather general circumstances. Hirschman and Widder [6] also discuss the behaviour of $F(y)$ as $y \rightarrow \pm \infty$ in great detail, refining the results of Haselgrove and Temperley in this direction.

Perhaps it is appropriate here to point out that $\Delta_{n}^{s} p_{A}(n)$ is positive for $s \leq k$ if $A$ has property $P_{k}$ as demonstrated by Bateman and Erdös [2].

We are greatly indebted to the referee who corrected many mistakes.

2. Estimates for $p_{A}(m, n)$ and $\bar{p}_{A}(m, n)$. We follow the approach of Haselgrove and Temperley very closely; however, the details are significantly altered so much of their proof will be repeated. We write 


$$
p_{A}(m, n)=\frac{1}{(2 \pi i)^{2}} \int_{|t|=e^{-\xi}} \int_{|u|=1} \frac{G(u, t)}{u^{m+1} t^{n+1}} d u d t
$$

(let $u=e^{i \varphi}=e^{-\alpha w}, t=e^{-w}$ )

$$
=\frac{1}{(2 \pi i)^{2}} \int_{\xi-i \pi}^{\xi+i \pi} \int_{-i \pi w^{-1}}^{i \pi w^{-1}} g(\alpha, w) \exp \{(m \alpha+n) w\} w d \alpha d w
$$

(let $w=\xi+i \theta)$

$$
\begin{array}{r}
=\frac{1}{4 \pi^{2} i} \int_{-\pi}^{\pi} \int_{-i \pi w^{-1}}^{i \pi w^{-1}} \exp \{\log g(\alpha, \xi+i \theta) \\
+(m \alpha+n)(\xi+i \theta)\} w d \alpha d \theta .
\end{array}
$$

In Richmond [8] it is shown that if $A$ satisfies (1.5) or (1.6) then $A$ has property II of [8], that is, with $\psi$ defined by $(1.9), A\left(x^{-1}\right)>\psi^{2 / 3+\eta}(x)$ as $x \rightarrow 0,1 / 2>\eta>0$ constant. We shall let $\eta$ have this value throughout the paper.

We shall divide the range of integration in (2.1) up as follows:

$A:|\theta| \leq \xi \delta \mu,|\alpha| \leq \mu$, where $\delta=\psi^{-1 / 2}(\xi), \mu=\psi^{(1-2 \eta) / 12}(\xi)$,

$B:|\theta| \leq \xi \delta \mu,|\alpha|>\mu$,

$C: \xi \mu \delta \leq|\theta| \leq \pi$.

On range $A$ we consider the double Taylor series expansion of the function $\log g(\alpha, \xi+i \theta)$ near $(0,0)$ :

$$
\begin{aligned}
\log g(\alpha, \xi+i \theta)= & -\sum \log \left(1-e^{-\left(a_{r}+\alpha\right)(\xi+i \theta)}\right) \\
= & -\sum \log \left(1-e^{-\left(a_{r}+\alpha\right) \xi}\right) \\
& -i \theta \sum\left(a_{r}+\alpha\right)\left(e^{\xi\left(a_{r}+\alpha\right)}-1\right)^{-1} \\
& +\frac{(i \theta)^{2}}{2} \sum\left(a_{r}+\alpha\right)^{2}\left(e^{\xi\left(a_{r}+\alpha\right)}-1\right)^{-2}+\ldots
\end{aligned}
$$

Let us consider the expansion in powers of $\alpha$ of

$$
\begin{aligned}
& \sum \log \left(1-e^{-\left(a_{r}+\alpha\right) \xi}\right) \\
& =\sum_{r} \log \left(1-e^{-\xi a_{r}}\right)+\alpha \sum \frac{\xi}{e^{\xi a_{r}}-1}-\frac{\alpha^{2}}{2} \sum \frac{\xi^{2} e^{\xi a_{r}}}{\left(e^{\xi a_{r}}-1\right)^{2}}+\ldots
\end{aligned}
$$

We write

$$
\sum \frac{\xi^{2} e^{-\xi a_{r}}}{\left(1-e^{-\xi a_{r}}\right)^{2}}=\sum \frac{1}{a_{r}^{2}} \frac{e^{-\xi a_{r}}}{\left(1-e^{-\xi a_{r}}\right)^{2}\left(\xi a_{r}\right)^{-2}}
$$




$$
\begin{aligned}
= & \sum_{a_{r} \leq \xi^{-3 / 4}} a_{r}^{-2}\left(1+O\left(\xi^{1 / 4}\right)\right) \\
& +O\left(\sum_{a_{r} \geq \xi^{-3 / 4}} \frac{\xi^{2}}{a_{r}^{2} \xi^{2}\left(1-e^{-\xi a_{r}}\right)^{2}}\right) \\
& \quad\left(\text { using } \exp (-x)=O\left(x^{-2}\right)\right) \\
= & \sum_{r=1}^{\infty} a_{r}^{-2}+O\left(\xi^{1 / 4}\right)+O\left(\sum_{a_{r} \geq \xi^{-3 / 4}} a_{r}^{-2} \xi^{-1 / 2}\right) \\
= & \sum_{r} a_{r}^{-2}+O\left(\xi^{1 / 4}\right) .
\end{aligned}
$$

Furthermore,

$$
\log K(\alpha)=-\sum_{r}\left(\log \left(1+\frac{\alpha}{a_{r}}\right)-\frac{\alpha}{a_{r}}\right)=\frac{\alpha^{2}}{2} \sum_{r} a_{r}^{-2}+O\left(\alpha^{3}\right) .
$$

We will use the following lemma proved by Haselgrove and Temperley [5]:

Lemma 2.1. If $w \rightarrow 0$ in a fixed Stolz angle $\Delta$ (i.e., $w \rightarrow 0$ in such a way that $|\operatorname{Im} w| \leq \Delta \operatorname{Re} w)$, and if $|\alpha| \leq \frac{3 \pi}{2} /\left(|w| \sqrt{1+\Delta^{2}}\right)$, then

$$
\log (K(\alpha) / g(\alpha, w))=o\left(|w|^{-2}\right)
$$

Also from Richmond [8], if $A$ satisfies (1.5) or (1.6) (so $A$ has properties (I) and (II) of [8]) then

$$
\sum a_{r}^{k}\left(e^{\xi a_{r}}-1\right)^{-l}=O\left(\xi^{-k} \psi^{1+\varepsilon}(\xi)\right), \quad \forall \varepsilon>0, k \geq l \geq 1,
$$

hence the coefficient of $\theta^{i} \alpha^{m}$ is $O\left(\xi^{-i} \psi^{1+\varepsilon}(\xi)\right)$. It follows from this estimate, (2.2) and Lemma 2.1 that

$$
\begin{aligned}
\log g(\alpha, \xi+i \theta)= & -\sum \log \left(1-e^{-\xi a_{r}}\right)-\alpha \xi \sum\left(e^{\xi a_{r}}-1\right)^{-1}+\log K(\alpha) \\
& -i \theta \sum a_{r}\left(e^{\xi a_{r}}-1\right)^{-1}-\frac{\theta^{2}}{2} \sum a_{r}^{2} e^{\xi a_{r}}\left(e^{\xi a_{r}}-1\right)^{-2} \\
& +O\left(\psi^{-1 / 4+\varepsilon}\right)
\end{aligned}
$$

over region $A$ (note $w=\xi+o(\xi)$ over $A$ ).

If we pick $\xi$ so that

$$
n=\sum a_{r}\left(e^{\xi a_{r}}-1\right)^{-1}=-\Psi^{\prime}(\xi)
$$

and define $m_{0}(\xi)$ as in Theorem 1.1 we have the integral

$$
\begin{array}{r}
\iint_{A} \exp \left\{-\sum \log \left(1-e^{-\xi a_{r}}\right)+\log K(\alpha)+\left(m-m_{0}\right) \alpha w+n \xi\right. \\
\left.-\frac{\theta^{2}}{2} \sum \frac{a_{r}^{2} e^{\xi a_{r}}}{\left(e^{\xi a_{r}}-1\right)^{2}}\right\}\left(1+O\left(\psi^{-1 / 4+\varepsilon}(\xi)\right)\right) w d \alpha d \theta .
\end{array}
$$


Note the $\alpha$ and $\theta$ variables are separated, so we consider

$$
\int_{-i|w| \mu / w}^{i|w| \mu / w} \exp \left\{\alpha w\left(m-m_{0}(\xi)\right)\right\} K(\alpha) d \alpha
$$

(recall that $\alpha w=-i \varphi$ so that $|\alpha| \leq \mu$ implies $|\varphi| \leq \mu|w|$ ).

Haselgrove and Temperley [5] also prove that

$$
|K(\alpha)|=O\left(|\alpha|^{-N}\right), \quad \forall N>0
$$

as $|\alpha| \rightarrow \infty$ with $|\operatorname{Re} \alpha|<1 / 2$.

Consider now the cases:

(i) $\left|m-m_{0}(\xi)\right| \xi \delta^{1 / 2} \leq 1$,

(ii) $\left|m-m_{0}(\xi)\right| \xi \delta^{1 / 2}>1$.

In case (i) we note that

$$
\left|m-m_{0}\right| \xi \delta \mu^{2} \leq \delta^{1 / 2} \mu^{2}=\psi^{-(1+4 \eta) / 12},
$$

hence

$$
\begin{aligned}
\left(m-m_{0}\right) w \alpha & =\left(m-m_{0}\right) \xi \alpha+O\left(\left(m-m_{0}\right) \xi \mu^{2} \delta\right) \\
& =\left(m-m_{0}\right) \xi \alpha+O\left(\psi^{-(1+2 \eta) / 12}\right) .
\end{aligned}
$$

Thus we can replace $w$ by $\xi$ in our integral. Also $w=\xi+i \theta=\xi+o(\xi)$ so

$$
\operatorname{Re}\left(\frac{i \mu|w|}{w}\right)=O\left(\frac{\xi \delta \mu \mu}{|w|}\right)=O\left(\psi^{-(1+\eta) / 3}\right) .
$$

From (2.7) and (2.9),

$$
\int_{-i|w| \mu / w}^{i|w| \mu / w} \exp \left\{\left(m-m_{0}\right) \xi \alpha\right\} K(\alpha) d \alpha=O(1) .
$$

Now as $\alpha$ goes from $i \mu|w| w^{-1}$ to $i \mu$ the $\operatorname{Re} \alpha$ goes from $\operatorname{Re}(i \mu|w| / w)$ to 0 . By (2.9), $\operatorname{Re}\left(\left(m-m_{0}\right) \xi \alpha\right)=O\left(\left(m-m_{0}\right) \xi \psi^{-(1+2 \eta) / 3}(\xi)\right)=o\left(\left(m-m_{0}\right) \xi \delta^{1 / 2}\right)=$ $o(1)$ in case (i) so by (2.7),

$$
\int_{i|w| \mu / w}^{i \mu} K(\alpha) \exp \left\{\left(m-m_{0}\right) \xi \alpha\right\} d \alpha=O\left(\mu^{-N}\right), \quad \forall N>0 .
$$

Again by (2.7),

$$
\int_{i|w| \mu / w}^{i \infty} K(\alpha) \exp \left\{\left(m-m_{0}\right) \xi \alpha\right\} d \alpha=O\left(\mu^{-N}\right), \quad \forall N>0 .
$$


Hence in case (i),

$$
\begin{aligned}
& \int_{-i \mu|w| / w}^{i \mu|w| / w} K(\alpha) \exp \left\{\left(m-m_{0}\right) \xi \alpha\right\} d \alpha \\
& =\int_{-i \infty}^{i \infty} K(\alpha) \exp \left\{\left(m-m_{0}\right) \xi \alpha\right\} d \alpha+O\left(\mu^{-N}\right), \quad \forall N>0 .
\end{aligned}
$$

In case (ii) we have $\left|m-m_{0}\right||w| \sim\left|m-m_{0}\right| \xi>\delta^{-1 / 2}$. Furthermore, one can deduce from Cauchy's integral formula (or by differentiating $\log K(\alpha)$ ) that $K^{\prime}(\alpha)=O\left(|\alpha|^{-N}\right), \forall N>0$ in the strip $|\operatorname{Re} \alpha|<1 / 4$. It now follows upon integrating by parts that

$$
\int_{-i \mu|w| / w}^{i \mu|w| / w} K(\alpha) \exp \left\{\left(m-m_{0}\right) \alpha w\right\} d \alpha=O\left(\delta^{1 / 2}\right) .
$$

Furthermore, from our estimate for $K^{\prime}(\alpha)$ we have $\int_{-i \infty}^{i \infty} K^{\prime}(\alpha) d \alpha=O(1)$ and integration by parts again shows that

$$
\int_{-i \infty}^{i \infty} K(\alpha) \exp \left\{\left(m-m_{0}\right) \xi \alpha\right\} d \alpha=O\left(\delta^{1 / 2}\right)+O\left(\psi^{-(1+2 \eta) / 12}\right) .
$$

Thus we can replace the finite limits in (2.11) by the limits in (2.12) and we conclude that in both cases (i) and (ii) we have, from (2.10),

$$
\begin{aligned}
& \int_{-i \mu|w| / w}^{i \mu|w| / w} K(\alpha) \exp \left\{\left(m-m_{0}\right) \alpha w\right\} d \alpha \\
= & \int_{-i \infty}^{i \infty} K(\alpha) \exp \left\{\left(m-m_{0}\right) \alpha \xi\right\} d \alpha+O\left(\delta^{1 / 2}\right)+O\left(\psi^{-(1+2 \eta) / 12}\right) .
\end{aligned}
$$

Also

$$
\begin{aligned}
& \frac{1}{2 \pi} \int_{-\mu \delta \xi}^{\mu \delta \xi} \exp \left\{\Psi(\xi)+n \xi-\frac{1}{2} \theta^{2} \Psi^{\prime \prime}(\xi)\right\} w d \theta \\
&=\frac{1}{\sqrt{2 \pi}} \xi\left(\Psi^{\prime \prime}(\xi)\right)^{-1 / 2} \exp \{\Psi(\xi)+n \xi\}\left(1+o\left(\psi^{-N}\right)\right) .
\end{aligned}
$$

Thus, for the $A$ part of range of integration in (2.1),

$$
\iint_{A}=\frac{1}{\sqrt{2 \pi}} \xi\left(\Psi^{\prime \prime}(\xi)\right)^{-1 / 2} \exp \{\Psi(\xi)+n \xi\}
$$


$\times\left\{\frac{1}{2 \pi i} \int_{-i \infty}^{i \infty} K(\alpha) \exp \left\{\left(m-m_{0}\right) \xi \alpha\right\} d \alpha+O\left(\psi^{(-1-2 \eta) / 12}(\xi)\right)+O\left(\psi^{-1 / 4}(\xi)\right)\right\}$.

We now consider the integral in (2.1) over the area $B$. Note that

$$
\frac{|G(u, t)|}{G\left(1, e^{-\xi}\right)}=\prod_{r} \frac{1-e^{-\xi a_{r}}}{\mid 1-e^{-\left(a_{r}+\alpha\right) w \mid}} .
$$

We write

$$
\beta=\arg \left(-\left(a_{r}+\alpha\right) w\right)=i \alpha w-a_{r} \theta
$$

since $\alpha w=-i \varphi$. Also $\operatorname{Re}\left(-a_{r} w\right)=-a_{r} \xi$ and hence

$$
\begin{aligned}
& \prod_{r} \frac{1-e^{-\xi a_{r}}}{\mid 1-e^{-a_{r} \xi+i \beta \mid}} \\
& \quad=\exp \left\{-\frac{1}{2} \sum \log \left(1+\frac{2 e^{\xi a_{r}}}{\left(e^{\xi a_{r}}-1\right)^{2}}(1-\cos \beta)\right)\right\} .
\end{aligned}
$$

Since $\beta=i \alpha w-a_{r} \theta=\varphi-a_{r} \theta$ we suppose $\left|a_{r} \theta\right| \leq 2$, so $|\beta| \leq \pi+2$ and hence

$$
1-\cos \beta \geq K \beta^{2}, \quad K>0 \text { a constant . }
$$

Furthermore, for $|\theta| \leq \xi \mu \delta, w=\xi+O(\xi \mu \delta)$ so $|\varphi|=|\alpha| \xi(1+O(\mu \delta))$. Hence unless $\left|a_{r} \theta\right| \leq \xi|\alpha| / 2$ we have $|\beta| \geq \xi|\alpha| / 2$. Let $\sum^{\prime}$ denote summation over $a_{r}$ such that $\left|a_{r} \theta\right| \leq \xi|\alpha| / 2 \leq \pi / 2, a_{r} \xi \leq 1$. Then by (2.18),

$$
\frac{|G(u, t)|}{G\left(1, e^{-\xi}\right)} \leq \exp \left\{-\frac{1}{2} \sum^{\prime} \log \left(1+\frac{e^{\xi a_{r}}}{2\left(e^{\xi a_{r}}-1\right)^{2}} K(\xi|\alpha|)^{2}\right)\right\}
$$

since each term in (2.16) is $\leq 1$ in absolute value. Also since $x \leq 1$ implies $\exp (-x) / 2(1-\exp (-x))^{2} \geq c x^{-2}$ this is

$$
\leq \exp \left\{-\frac{1}{2} \sum^{\prime} \log \left(1+K c|\alpha|^{2} a_{r}^{-2}\right)\right\} \text {. }
$$

If $a_{r} \leq(K c)^{1 / 2}|\alpha|^{1 / 2}$ then $a_{r} \theta=O\left(\xi \mu \delta|\alpha|^{1 / 2}\right)=o\left(\xi|\alpha|^{1 / 2}\right)$. Hence

$$
\begin{aligned}
& \frac{|G(u, t)|}{G\left(1, e^{-\xi}\right)} \\
& \quad \leq \exp \left\{-\frac{1}{2} A\left(|\alpha|^{1 / 2}(K c)^{1 / 2}\right) \log |\alpha|\right\}<|\alpha|^{-N}, \quad \forall N>0 .
\end{aligned}
$$

Now $|\theta| \leq \mu \xi \delta$ and $w=\xi(1+o(\mu \delta))$ so from (2.19),

$$
\iint_{B}=O\left(\mu \xi^{2} \delta|t|^{-n} G\left(1, e^{-\xi}\right) \mu^{-N}\right), \quad \forall N>0 .
$$

Now

$$
\Psi^{\prime \prime}(\xi)=\sum a_{r}^{2} e^{\xi a_{r}}\left(e^{\xi a_{r}}-1\right)^{-2}=O\left(\xi^{-2} \psi^{1+\varepsilon}(\xi)\right)
$$


since $A$ satisfies (1.5) or (1.6). Hence $\left(\Psi^{\prime \prime}(\xi)\right)^{-1 / 2} \geq c \xi \psi^{-1 / 2-\varepsilon}(\xi)=c \xi \delta \psi^{-\varepsilon}$ and from (2.20) we obtain

$$
\iint_{B}=O\left(\xi\left(\Psi^{\prime \prime}(\xi)\right)^{-1 / 2} \exp \{\Psi(\xi)+n \xi\} \mu^{-N}\right), \quad \forall N>0 .
$$

Finally, we consider the integral in (2.1) over area $C$. Following Richmond [8] we suppose that the range $\xi \mu \delta \leq \theta \leq \pi$ is divided into three ranges, $I^{\prime}=[\xi \mu \delta, \xi], I^{\prime \prime}=\left[\xi, \xi \psi^{c}(\xi)\right]$ and $I^{\prime \prime \prime}=\left[\xi \psi^{c}(\xi), \pi\right]$. We use (2.17) and recall that $\beta=\varphi-a_{r} \theta$. Note that if $a_{r} \leq|\alpha|^{1 / 2}$ then $a_{r} \theta \leq \xi|\alpha|^{1 / 2}$ and so $|\beta| \geq \xi|\alpha| / 2$. There will be at least $A\left(|\alpha|^{1 / 2}\right)$ such $a_{r}$ and the arguments leading to $(2.19)$ give

$$
\frac{|G(u, t)|}{G\left(1, e^{-\xi}\right)} \leq \exp \left\{-\frac{1}{2} A\left(|\alpha|^{1 / 2}\right) \log \left(1+\frac{K|\alpha|^{2}}{|\alpha|}\right)\right\} \leq|\alpha|^{-N}, \quad N>0,
$$

and hence the arguments leading to (2.21) now give

$$
\begin{aligned}
\int_{I^{\prime}} & \int_{|\alpha| \geq \mu^{1 / 2}} d \alpha d \theta \\
& =O\left(\xi\left(\Psi^{\prime \prime}(\xi)\right)^{-1 / 2} \exp \{\Psi(\xi)+n \xi\} \mu^{-N}\right), \quad \forall N>0 .
\end{aligned}
$$

If $|\alpha| \leq \mu^{1 / 2}$ we consider those $a_{r}$ such that $a_{r} \xi \leq 1$. If $\left|a_{r} \theta\right| \geq 2|\varphi|$ then $\left|\varphi-a_{r} \theta\right| \geq a_{r}|\theta| / 2$. Hence from (2.17),

$$
\begin{aligned}
\frac{|G(u, t)|}{G\left(1, e^{-\xi}\right)} & \leq \exp \left\{-\frac{1}{2} \sum_{a_{r} \geq 2|\varphi||\theta|^{-1}} \xi^{-1} \log \left(1+\frac{K c a_{r}^{2} \theta^{2}}{\xi^{2} a_{r}^{2}}\right)\right\} \\
& \leq \exp \left\{-\frac{1}{4} \sum_{a_{r} \geq 2|\varphi||\theta|^{-1}} \xi^{-1} K c \mu^{2} \delta^{2}\right\} .
\end{aligned}
$$

Since $|\varphi|=|\alpha||w| \leq 2|\alpha| \xi \leq 2 \mu^{1 / 2} \xi$ and $\theta \geq \xi \mu \delta$ this is

$$
\leq \exp \left\{-\frac{K c}{4}\left(A\left(\xi^{-1}\right)-A\left(4 \delta^{-1} \mu^{-1 / 2}\right)\right) \mu^{2} \delta^{2}\right\} .
$$

Now by the discussion following (2.1), we have $A\left(\xi^{-1}\right) \geq \psi^{2 / 3+\eta}(\xi)$ and $A\left(4 \delta^{-1} \mu^{-1 / 2}\right)<\delta^{-1}=\psi^{1 / 2}(\xi)$ so this is

$$
\leq \exp \left\{-c^{\prime} \psi^{2 / 3+\eta} \psi^{-5 / 6-\eta / 3}\right\}=\exp \left\{-c^{\prime} \psi^{(4 \eta-1) / 6}(\xi)\right\} .
$$

If $A$ satisfies (1.5) or (1.6) then Lemma 2.5 of [8] and the proof of Lemma 3.3 of [8] yield that $\eta>1 / 4$.

Thus

$$
\int_{|\alpha| \leq \mu^{1 / 2}} \int_{I^{\prime}} d \theta d \alpha=O\left(\xi \mu^{1 / 2} \xi \exp \{\Psi(\xi)+n \xi\} \exp \left\{-c^{\prime} \psi^{(4 \eta-1) / 6}(\xi)\right\}\right.
$$


Now from this and (2.22) it follows that

$$
\begin{aligned}
& \int_{I^{\prime}} \int_{-i \pi w^{-1}}^{i \pi w^{-1}} d \alpha d \theta \\
& \quad=O\left(\xi\left(\Psi^{\prime \prime}(\xi)\right)^{-1 / 2} \exp \{\Psi(\xi)+n \xi\} \mu^{-N}\right), \quad \forall N>0 .
\end{aligned}
$$

We now consider the integration in (2.1) over $C$ with $\theta \in I^{\prime \prime}$. Again we consider the cases $|\alpha| \geq \xi \mu^{1 / 2}$ and $|\alpha| \leq \mu^{1 / 2}$. If $a_{r} \leq|\alpha|^{1 / 2}$ and $c<$ $(1-2 \eta) / 48$ then $a_{r} \theta \leq O\left(\xi|\alpha|^{1 / 2} \psi^{c}(\xi)\right)$ on $I^{\prime \prime}$ so $a_{r} \theta=o(\xi|\alpha|)$ if $|\alpha| \geq \mu^{1 / 2}$. Now $|\varphi|=|\alpha||w| \geq \xi|\alpha|$ so the derivation of (2.21) gives

$$
\begin{aligned}
\int_{I^{\prime \prime}} & \int_{|\alpha| \geq \mu^{1 / 2}} d \alpha d \theta \\
& =O\left(\xi\left(\Psi^{\prime \prime}(\xi)\right)^{-1 / 2} \exp \{\Psi(\xi)+n \xi\} \mu^{-N}\right), \quad \forall N>0 .
\end{aligned}
$$

If $|\alpha| \leq \mu^{1 / 2}$ we follow the derivation after (2.22) and obtain

$$
\begin{aligned}
\frac{|G(u, t)|}{G\left(1, e^{-\xi}\right)} & \leq \exp \left\{-\frac{1}{2} \sum_{a_{r} \geq 2|\varphi| \theta^{-1}}^{\xi^{-1} \psi^{-c}} \log (1+c)\right\} \\
& <\exp \left\{-K\left(A\left(\xi^{-1} \psi^{-c}(\xi)-A\left(3 \mu^{1 / 2}\right)\right)\right)\right\} .
\end{aligned}
$$

An examination of the proof of Lemma 3.3 of [8] shows that when $A$ satisfies (1.5) or (1.6) we can find a $c$ such that $A\left(\xi^{-1} \psi^{-c}(\xi)\right)>\psi^{1 / 2+\varepsilon}(\xi)$. Since $A\left(3 \mu^{1 / 2}\right) \leq 3 \mu^{1 / 2}$ we get $\leq \mu^{-N}, \forall N>0$. The argument leading to (2.23) now gives

$$
\begin{aligned}
\int_{I^{\prime \prime}} & \int_{|\alpha| \leq \mu^{1 / 2}} d \alpha d \theta \\
& =O\left(\xi\left(\Psi^{\prime \prime}(\xi)\right)^{-1 / 2} \exp \{\Psi(\xi)+n \xi\} \mu^{-N}\right), \quad \forall N>0 .
\end{aligned}
$$

Equations (2.24) and (2.25) now give

$$
\begin{aligned}
& \int_{I^{\prime \prime}} \int_{-i \pi w^{-1}}^{i \pi w^{-1}} d \alpha d \theta \\
& \quad=O\left(\xi\left(\Psi^{\prime \prime}(\xi)\right)^{-1 / 2} \exp \{\Psi(\xi)+n \xi\} \mu^{-N}\right), \quad \forall N>0 .
\end{aligned}
$$

We now use the following lemma from Richmond [8]. We write $2 \pi \varphi$ resp. $2 \pi \theta$ instead of $\varphi$ resp. $\theta$.

Lemma 2.2. Let $c$ be any constant with $0<c<1 / 2$. If $\varphi-a_{r} \theta \in[c, 1-c]$ then

$$
\frac{1-e^{-\xi a_{r}}}{\mid 1-e^{-\xi a_{r}+2 \pi i\left(\varphi-a_{r} \theta\right) \mid}} \leq(1-\cos c)^{-1 / 2}\left(1-e^{-\xi a_{r}}\right)\left(1+e^{-2 \xi a_{r}}\right)^{-1 / 2} .
$$


Lemma 2.3. Let $N$ be an arbitrary integer. Let $c$ be any constant with $0<c<1$. There is a constant $\theta_{0}=\theta_{0}(N)>0$ such that if $\theta \in\left[\xi \psi^{c}(\xi), \theta_{0}\right]$ then

Proof. If

$$
\frac{|G(u, t)|}{G\left(1, e^{-\xi}\right)}=O\left(\psi^{-N}(\xi)\right)
$$

$$
\theta \notin\left[\frac{\varphi+l}{a_{r}}-\xi \psi^{c}(\xi), \frac{\varphi+l}{a_{r}}+\xi \psi^{c}(\xi)\right], \quad l=0,1, \ldots, a_{r}-1,
$$

then from Lemma 2.2,

Suppose

$$
\frac{1-e^{-\xi a_{r}}}{\mid 1-e^{-\xi a_{r}+2 \pi i\left(\varphi-a_{r} \theta\right) \mid}}=O\left(\psi^{-c}(\xi)\right)
$$

$$
\frac{\varphi+l}{a_{1}}=\frac{\varphi+m}{a_{2}} \quad \text { or } \quad \varphi=\frac{a_{1} m-a_{2} l}{a_{2}-a_{1}} .
$$

If $\varphi \neq 0$ then $|\varphi| \geq\left(a_{2}-1\right)^{-1}$. We find that if $0<|\varphi|<\left(a_{2}-1\right)^{-1}$ then, for $|\theta| \leq\left(3 a_{2}\right)^{-1}$,

$$
\frac{\left(1-e^{-\xi a_{1}}\right)\left(1-e^{-\xi a_{2}}\right)}{\left|\left(1-e^{-\xi a_{1}+2 \pi i\left(\varphi-a_{1} \theta\right)}\right)\right| \mid 1-e^{-\xi a_{2}+2 \pi i\left(\varphi-a_{2} \theta\right) \mid}}=O\left(\psi^{-c}(\xi)\right) .
$$

We can repeat this argument for the pair $a_{3}$ and $a_{4}$ and so on. We conclude that if $0<|\varphi|<\left(a_{I}-1\right)^{-1}, I=2[N / c]+2,|\theta| \leq\left(3 a_{I}\right)^{-1}$

$$
\frac{|G(u, t)|}{G\left(1, e^{-\xi}\right)}=O\left(\psi^{-N}(\xi)\right) \text {. }
$$

Furthermore, if $|\varphi| \geq\left(a_{I}-1\right)^{-1}, I=2[N / c]+2$ and $a_{r} \theta \leq\left(a_{I}-1\right)^{-1} / 2$ then $\left|\varphi-a_{r} \theta\right| \geq\left(a_{I}-1\right)^{-1} / 2$.

Clearly we can choose a $\theta_{1}$ so that if $|\theta| \leq \theta_{1}$ then $\left|a_{r} \theta\right| \leq\left(a_{I}-1\right)^{-1} / 2$ for $r=1, \ldots,[N / c]+1$. Again from Lemma 2.2 such a choice of $\theta_{1}$ gives

$$
\frac{|G(u, t)|}{G\left(1, e^{-\xi}\right)}=O\left(\psi^{-N}(\xi)\right) \text {. }
$$

Finally, if $\varphi=0$ we can choose a $\theta_{2}$ so that $|\theta| \leq \theta_{2}$ implies $\left|a_{r} \theta\right| \leq 1 / 2$ for $r=1, \ldots,[N / c]+1$ and again Lemma 2.2 implies

$$
\frac{|G(u, t)|}{G\left(1, e^{-\xi}\right)}=O\left(\psi^{-N}(\xi)\right) \text {. }
$$

Lemma 2.3 follows from $(2.27),(2.28)$ and $(2.29)$ with $\theta_{0}=\min \left(\theta_{1}, \theta_{2}\right)$.

We now follow Richmond [10] to estimate $G(u, t)$ for $\theta_{0} \leq|\theta| \leq 1 / 2$.

Lemma 2.4. If $\theta_{0} \leq|\theta| \leq 1 / 2$ then

$$
\frac{|G(u, t)|}{G\left(1, e^{-\xi}\right)}=O\left(\xi^{k+l+2}\right)+O\left(\xi^{3+k} \mu^{2} \delta\right) .
$$


Pr o of. Recall the definition of $\bar{A}$ preceding (1.10). If

$$
\theta \notin[-\delta, \delta] \cup \bigcup_{a \in \bar{A}} \bigcup_{|l|=0}^{a}\left[\frac{\varphi+l}{a}-\delta, \frac{\varphi+l}{a}+\delta\right],
$$

then from Lemma 2.2,

$$
\prod_{a \in \bar{A}} \frac{1-\exp (-\xi a)}{1-\exp \{-\xi a+2 \pi i(\varphi-a \theta)\}}=O\left(\xi^{k+1}\right) .
$$

Suppose now $\theta \in[-\delta+(\varphi+l) / a, \delta+(\varphi+l) / a]$. Suppose $\varphi-b \theta=\varphi-a \theta-l$, $l \in \mathbb{Z}$, or $(b-a) \theta=l$. Since $|\theta| \leq 1 / 2$ we may suppose $|l| \leq|b-a| / 2$. Suppose this relation holds for all $b \in A$. Then $l\left(b_{1}-a\right)=l_{1}(b-a)$ so

$$
\frac{l_{1}}{\operatorname{gcd}\left(l_{1}, b_{1}-a\right)}(b-a)=\frac{l\left(b_{1}-a\right)}{\operatorname{gcd}\left(l_{1}, b_{1}-a\right)} .
$$

Hence if $\Delta=\left(b_{1}-a\right) / \operatorname{gcd}\left(l_{1}, b_{1}-a\right)$ then $\Delta>1$ and $\Delta \mid b-a$ for each $b \in A$. Thus $A$ does not have property $Q_{0}$. Hence we can find a $b$ so that $\varphi-b \theta \neq \varphi-a \theta+l, l \in \mathbb{Z}$. If $A$ has property $Q_{k}$ then deleting an element leaves us with a set having property $Q_{k-1}$. Hence we can find a set $A_{a}$ consisting of $k+1$ such $b$ 's. Hence

$$
\prod_{a \in A_{a}} \frac{1-\exp (-\xi a)}{1-\exp \{-\xi a+2 \pi i(\varphi-a \theta)\}}=O\left(\xi^{k+1}\right)
$$

for these $\theta$.

From (2.30) and (2.31), with $\widetilde{A}=\bar{A} \cup \bigcup_{a \in \bar{A}} A_{a}$, and $\theta \notin[-\delta, \delta]$, we have

$$
\prod_{a \in \widetilde{A}} \frac{1-\exp (-\xi a)}{1-\exp \{-\xi a+2 \pi i(\varphi-a \theta)\}}=O\left(\xi^{k+1}\right) .
$$

Now every element of $A-\widetilde{A}$ is congruent to $r$ modulo $d$ (as defined in (1.10)), i.e., $a=m d+r$ for $a \in A-\widetilde{A}$. Let $B$ denote the sequence of $m$ 's defined in this way and let $\widetilde{B}$ be defined as $\widetilde{A}$. The argument giving (2.32) gives

$$
\prod_{m \in \widetilde{B}} \frac{1-\exp \{-\xi(m d+r)\}}{1-\exp \{-\xi(m d+r)+2 \pi i((\varphi-r \theta)-m d \theta)\}}=O\left(\xi^{l+1}\right)
$$

provided $\|d \theta\| \geq c$ where $\|x\|=$ the distance of $x$ from the nearest integer and $c$ is a positive constant. Equations (2.32) and (2.33) show the existence of a $\delta$ such that

$$
\frac{|G(u, t)|}{G\left(1, e^{-\xi}\right)}=O\left(\xi^{k+l+2}\right)
$$


unless

$$
\theta \in[-\delta, \delta] \cup \bigcup_{|l|=1}^{d-1}[l / d-\delta, l / d+\delta]
$$

Let

$$
I_{1}=[l / d-\delta, l / d+\delta] \quad \text { and } \quad I_{1}^{1}=[l / d-\xi \mu \delta, l / d+\xi \mu \delta] .
$$

The proofs of (2.23) and (2.26) and of Lemma 2.3 give

$$
\int_{\theta \in I_{1}-I_{1}^{1}} \int_{-i \pi / w}^{i \pi / w} d \alpha d \theta=O\left(\xi\left(\Psi^{\prime \prime}(\xi)\right)^{-1 / 2} \exp \{\Psi(\xi)+n \xi\} \psi^{-N}\right) .
$$

If $\theta \in I_{1}^{1}$, however, the proof of (2.21) shows that

$$
\int_{\theta \in I_{1}^{1}} \int_{|\varphi-r l / d| \geq \xi \mu} d \alpha d \theta=O\left(\xi\left(\Psi^{\prime \prime}(\xi)\right)^{-1 / 2} \exp \{\Psi(\xi)+n \xi\} \psi^{-N}(\xi)\right) .
$$

However, from (2.32),

$$
\int_{\theta \in I_{1}^{1}} \int_{|\varphi-r l / d| \leq \xi \mu} d \varphi d \theta=O\left(\xi^{k+3} \mu^{2} \delta\right) .
$$

This proves Lemma 2.4 .

Now from Richmond [8] we have $\Psi^{\prime \prime}(\xi)=O\left(\xi^{-2} \psi^{1+\varepsilon}(\xi)\right)$. Also $\psi(\xi) \leq$ $\sum_{r} \exp (-\xi r)=O\left(\xi^{-1}\right)$. Hence

$$
\xi\left(\Psi^{\prime \prime}(\xi)\right)^{-1 / 2} \geq \xi^{5 / 2+\varepsilon} .
$$

Now Theorem 1.1 follows when $r=s=0$ from (2.14), (2.15), (2.21), (2.23), (2.26), Lemmas 2.3 and 2.4, and (2.38). If $A$ has property $Q_{k}$ for all $k$ then we get the result for all $r$ and $s$ by considering, as do Haselgrove and Temperley [5],

$$
\frac{1}{2 \pi i} \iint \frac{G(x, z)}{x^{m+1} z^{n+1}}(1-z)^{s}(1-x)^{r} d x d z .
$$

Note finally that Theorem 1.2 now follows easily since the generating function for $\bar{p}_{A}(m, n)$ is $(1-z)^{-1} G(x, z)$.

3. Examples and further discussion. The moment generating function of $F(y)$ is, as we have seen,

$$
K(\alpha)=\prod_{r=1}^{\infty}\left(1+\frac{\alpha}{a_{r}}\right)^{-1} e^{\alpha / a_{r}} .
$$

Hence the mean of $F(y)$ is at $y=0$ or equivalently $m=m_{0}(\xi)$. 
Since

$m_{0}(\xi)=\sum_{r}\left(e^{\xi a_{r}}-1\right)^{-1}=\sum_{r} \frac{1}{\xi a_{r}} \cdot \frac{e^{-\xi a_{r}}}{\left(1-e^{-\xi a_{r}}\right)\left(\xi a_{r}\right)^{-1}}>c \xi^{-1} \sum_{a_{r} \leq \xi^{-1}} a_{r}^{-1}$,

we see that if $\sum a_{r}^{-1}$ diverges then for almost all of the partitions counted by $p_{A}(n)$ we have

$$
m \sim m_{0}(\xi)
$$

when either (1.5) or (1.6) holds and $k+l \geq 1$ in Theorem 1.1. We also conclude from Theorem 1.2 that (3.1) holds for almost all of the partitions counted by $\bar{p}_{A}(n)$ when $\sum a_{r}^{-1}$ diverges, $Q_{0}$ and either (1.5) or (1.6) holds. It is interesting that (3.1) answers the question posed by Turán [15] for questions I and III. Note also that Erdős and Turán [4] did not need an arithmetical condition in their solution for $\bar{q}_{A}(n)$.

If $\sum a_{r}^{-1}<\infty$ then we can say that the number of partitions with $m$ in the range $m_{0}+y_{1} \xi^{-1} \leq m \leq m_{0}+y_{2} \xi^{-1}$ is

$$
\sim p_{A}(n) \int_{y_{1}}^{y_{2}} F(y)
$$

when the assumptions of Theorem 1.1 hold and $k+l \geq 1$. We can say in this case that almost all of the partitions counted by $p_{A}(n)$ have $m \leq m_{0}(\xi)+$ $w(n) \xi^{-1}$ where $w(n)$ is any function such that $w(n) \rightarrow \infty$ as $n \rightarrow \infty$. It is well known that (see Hirschman and Widder [6]) $F(y)=0$ for $y \leq-\sum a_{r}^{-1}$. While we have a rather complete understanding of the case $\sum a_{r}^{-1}<\infty$ by using Theorems 1.1 and 1.2 we will not have (3.1) holding.

We now consider the case when $A$ is the set of primes, i.e., $a_{r}=$ the $r$ th prime. It is easily verified from the prime number theorem that both (1.5) and (1.6) hold. $A$ has property $Q_{0}$ and deleting 2 leaves us with a sequence of odd numbers, deleting any other prime does not leave us with an arithmetic progression. Thus $r=1$ and $d=2$ in (1.10) and the sequence $B$ has property $Q_{l}$ for all $l \geq 0$. Hence Theorem 1.1 applies to give us the asymptotic formula for $p_{A}(m, n)$ found by Haselgrove and Temperley [5].

\section{References}

[1] F. C. Auluck, S. Chowla and H. Gupta, On the maximum value of the number of partitions of $n$ into $k$ parts, J. Indian Math. Soc. (N.S.) 6 (1942), 105-112.

[2] P. Bateman and P. Erdős, Monotonicity of partition functions, Mathematika 3 (1956), 1-14.

[3] P. Erdös and J. Lehner, The distribution of the number of summands in the partitions of a positive integer, Duke Math. J. 8 (1941), 335-345.

[4] P. Erdős and P. Turán, On some general problems in the theory of partitions, I, Acta Arith. 18 (1971), 53-62. 
[5] C. B. Haselgrove and H. N. V. Temperley, Asymptotic formulae in the theory of partitions, Proc. Cambridge Philos. Soc. 50 (1954), 225-241.

[6] I. I. Hirschman and D. V. Widder, The Convolution Transform, Princeton University Press, Princeton, 1955.

[7] G. Meinardus, Asymptotische Aussagen über Partitionen, Math. Z. 59 (1954), 388-398.

[8] L. B. Richmond, Asymptotic relations for partitions, J. Number Theory 7 (1975), 389-405.

[9] —, The moments of partitions, II, Acta Arith. 28 (1975), 229-243.

[10] - Asymptotic relations for partitions, Trans. Amer. Math. Soc. 219 (1976), 379385.

[11] K. F. Roth and G. Szekeres, Some asymptotic formulae in the theory of partitions, Quart. J. Math. Oxford Ser. (2) 5 (1954), 241-259.

[12] W. Schwarz, Schwache asymptotische Eigenschaften von Partitionen, J. Reine Angew. Math. 232 (1968), 1-16.

[13] G. Szekeres, An asymptotic formula in the theory of partitions, I, Quart. J. Math. Oxford Ser. (2) 2 (1951), 85-108.

[14] - Some asymptotic formulae in the theory of partitions, II, ibid. 4 (1953), 96-111.

[15] P. Turán, Combinatorics, partitions, group theory, in: Proc. Internat. Colloq. in Combin. Theory, Accad. Naz. Lincei, Rome, 1973, T. II, 1976, 181-200.

DEPARTMENT OF COMBINATORICS AND OPTIMIZATION

UNIVERSITY OF WATERLOO

WATERLOO, ONTARIO

CANADA N2L 3G1

Received on 13.5.1992

and in revised form on 21.12.1993 